\title{
Multi-actuated mechanism design considering structure flexibility using correlated performance reinforcing"
}

\author{
Jing-hua XU, Shu-you ZHANG ${ }^{\dagger \ddagger}$, Jian-rong TAN, Zhen ZHAO \\ (The State Key Laboratory of Fluid Power Transmission and Control, Zhejiang University, Hangzhou 310027, China) \\ †E-mail: zsy@zju.edu.cn
}

Received Dec. 28, 2014; Revision accepted Aug. 24, 2015; Crosschecked Oct. 13, 2015

\begin{abstract}
Multi-actuated rigid-flexible dynamic system exists widely in precision machinery and electrical control fields. The performances, such as kinematic, dynamic, electrical, magnetic, and thermal performances, are correlated and difficult to trap precisely. Therefore, a multi-actuated mechanism design method considering structure flexibility using correlated performance reinforcing is put forward. A system containing flexible subparts with multi degrees of freedom (DOF) with physical coordinate is converted into modal coordinate using 'DOF $\times$ modal order' square matrix. The structure flexibility is described by modal superposition of the shape mode which is considered as additional generalized coordinates. A dynamic equation with large DOF is formulated and reduced based on Craig-Bampton modal truncation. Using analogical design methodology with and without structure flexibility of the low voltage circuit breaker (LVCB), the extent of the performance impact of each subpart is obtained by calculating correlated Holm force, Lorentz force, electrodynamic repulsion force, electromagnetic force, and cantilevered bimetallic strip force. Design of experiments method is employed to reveal the hard-measuring properties using correlated relatively easy-measuring parameters. The trip mechanism is validated by an electrical performance experiment. results show that the structure flexibility can decrease the tripping velocity, which is non-negligible, especially for high frequency tripping. The method provides a reference significance for similar multi-actuated mechanism design.
\end{abstract}

Key words: Multi-actuated mechanism, Electro-thermal-magnetic mechanism, Multi-body rigid-flexible dynamic system, Low voltage circuit breaker (LVCB), Correlated performance reinforcing

doi: $10.1631 /$ jzus.A1500003

Document code: A

CLC number: TM561

\section{Introduction}

Low voltage circuit breaker (LVCB) is extremely widely used in electric power distribution branches to protect electrical circuits from damage by tripping in instantaneous, short-time delay, and

\footnotetext{
¿ Corresponding author

* Project supported by the National Basic Research Program (973 Program) of China (No. 2011CB706506), the National Natural Science Foundation of China (Nos. 51221004 and 51375012), the National High-Tech R\&D Program (863 Program) of China (Nos. 2013AA041303 and 2013IM030500), and the Zhejiang Provincial Natural Science Foundation of China (No. Y13E050014)

(iD) ORCID: Jing-hua XU, http://orcid.org/0000-0001-8503-8515; Shu-you ZHANG, http://orcid.org/0000-0001-9023-5361

(C) Zhejiang University and Springer-Verlag Berlin Heidelberg 2015
}

long-time delay styles in case of a short circuit, overload, under voltage, phase failure or leakage. Moulded case circuit breaker (MCCB) is a typical LVCB with instantaneous, short-time delay, and long-time delay function using the trip mechanism with electrodynamic force, electromagnetic force, bimetallic strip force, and spring force. Obviously, trip mechanism is the key functional module of a LVCB.

Research on circuit breakers (Lee and Kim, 2006; Li et al., 2007; Braun and Goldfarb, 2012; Hesse and Palacios, 2012; Machado et al., 2012; Deb and Sen, 2013) has focused mainly on mechanism synthesis, interruption process, and shape optimization. A trip mechanism actuated by thermal and magnetic force commonly has the metamorphic 
property that the effective number of degrees of freedom (DOFs) changes with variable topology. Nossov et al. (2007) studied simulation of the thermal radiation effect of an arc on polymer walls in LVCBs, taking into account heating, volume thermal decomposition, and fusion of the polymer, as well as the screening action of the vapor from the surface. Lu et al. (2007) studied the instantaneous protection reliability of LVCBs, described their main failure modes, and gave the method of eliminating the non-periodic component of test current. Razi-Kazemi (2015) proposed a prevalent and conventional diagnosis method of the mechanical operations (called timing test) through the travel curve (TC) to reveal the correlation between the TC and auxiliary contacts of the LVCB.

From the perspective of basic theory of LVCB, many scholars studied the basic multi-body dynamics. Nikravesh (2007) researched the initial condition correction in multi-body dynamics. The trip mechanism makes the trapping of correlated performance difficult, especially with flexible bodies. However, flexible bodies can contribute large amounts of high frequency components which may need a prohibitively small iteration step. What is more, electromechanical coupling is found widely in LVCB whose actuating domains are complex and overlapping. Therefore, the rigid-flexible multi-body dynamics are very complicated and difficult to simulate and trap.

The difficulty of multi-actuated mechanism design lies in the electromechanical coupling found in different actuating processes based on our previous work in performance design (Xu et al., 2013a; 2013b). To study this phenomenon, we proposed a multi-actuated mechanism design method considering structure flexibility using correlated performance reinforcing.

\section{Modal coordinates expression of rigid- flexible multi-DOF multi-body system}

DOFs are a measure of how components can move relative to one another in a model. A body free in Cartesian space has six DOFs in which it can move: three translational $x, y$, and $z$, and three rotational $\alpha, \beta$, and $\gamma$. The orientation of a body in 3D Euclidean space can be represented by Euler angles, namely, precession angle $\alpha$, nutation angle $\beta$, and self-rotation angle $\gamma$. Each DOF corresponds to at least one equation of motion. The Euler angles are generalized coordinates of a flexible body.

Obviously, each mass point has zero or more DOFs. A system with $n$ DOFs has less than $n$ mass points once each mass point has more than one DOF. A system or structure that has $n$ DOFs has $n$ inherent frequencies, $n$ order modals, and $n$ modal vectors (Fig. 1). The modal vector of $n$ DOFs system can be denoted by 'DOF $\times$ modal order' square matrix. For $n$ order square matrix $(n \times n)$, it has $n$ eigenvalues and $n$ eigenvectors. Eigenvalues are the quadratic of inherent frequency. Eigenvectors are mode shapes. Each mode has specific resonant frequencies, mode damping, mode shapes, mode stiffness, and mode mass, and represents the response of all DOFs.

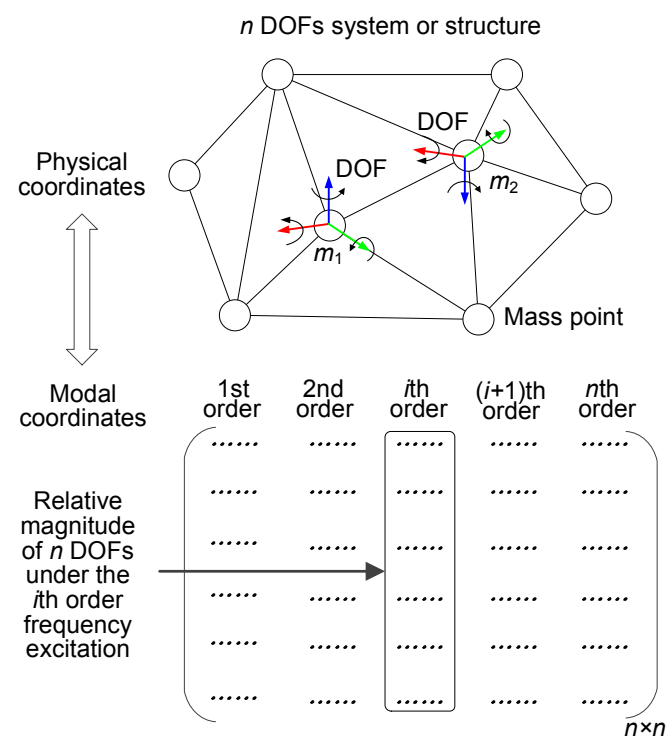

Fig. 1 Modal vector of an $n$ DOFs system or structure

A rigid body is not deformable. By contrast, a flexible body is deformable regardless of elastic deformation or plastic deformation. The inherent or natural frequency of a rigid body is zero. A rigid body has only a rigid modal, but a flexible body contains $n$ $(n=1,2, \ldots)$ modals.

Modal analysis includes freedom modal analysis and constraint modal analysis, each of which is used to approximate the actual problems. The size of a finite element (FE) model can be reduced on the 
premise that the inherent frequency is larger than the external excitation frequency. The Craig-Bampton method (C-B method) is used for reducing the size of a model (Kim et al., 2014). The C-B method combines the motion of boundary points with modes of the structure, assuming the boundary points are held fixed. The method is similar to other reduction schemes, such as Guyan reduction and modal decoupling. Modal truncation means considering major modal coordinates and ignoring minor coordinates. For each substructure, the DOF $\boldsymbol{u}$ are partitioned into boundary DOF $\left(\boldsymbol{u}_{\mathrm{b}}\right)$ and interior DOF $\left(\boldsymbol{u}_{\mathrm{i}}\right)$ :

$$
\boldsymbol{u}=\left\{\begin{array}{l}
\boldsymbol{u}_{\mathrm{b}} \\
\boldsymbol{u}_{\mathrm{i}}
\end{array}\right\}=\left(\begin{array}{cc}
\boldsymbol{I} & 0 \\
\boldsymbol{\Phi}_{\mathrm{ic}} & \boldsymbol{\Phi}_{\mathrm{in}}
\end{array}\right)\left(\begin{array}{l}
\boldsymbol{q}_{\mathrm{c}} \\
\boldsymbol{q}_{\mathrm{n}}
\end{array}\right),
$$

where $\boldsymbol{\Phi}_{\text {ic }}$ and $\boldsymbol{\Phi}_{\text {in }}$ are the physical displacements of the interior DOF in the constraint and normal modes, respectively; and $\boldsymbol{q}_{\mathrm{c}}$ and $\boldsymbol{q}_{\mathrm{n}}$ are the modal coordinates of the constraint and fixed-boundary normal modes, respectively; $\boldsymbol{I}$ is identity matrix.

The generalized (caret $\wedge$ ) stiffness and mass matrix corresponding to the Craig-Bampton modal basis are obtained via a modal transformation. The generalized stiffness transformation is block diagonal:

$$
\hat{\boldsymbol{K}}=\boldsymbol{\Phi}_{s}^{\mathrm{T}} \boldsymbol{K} \boldsymbol{\Phi}_{s}=\operatorname{diag}\left(\omega_{s}\right)
$$

The generalized mass transformation is

$$
\hat{\boldsymbol{M}}=\boldsymbol{\Phi}_{s}^{\mathrm{T}} \boldsymbol{M} \boldsymbol{\Phi}_{s},
$$

where $\boldsymbol{\Phi}_{s}$ is a generalized modal of the first $s$ order frequency $\omega_{s}$.

The eigenvectors are arranged in a transformation matrix $\boldsymbol{\Phi}_{s}$, which transforms the CraigBampton modal basis to an equivalent, orthogonal basis with modal coordinates $\boldsymbol{q}^{*}$ :

$$
\boldsymbol{\Phi}_{s} \boldsymbol{q}^{*}=\boldsymbol{q}
$$

The DOF superposition formula is

$$
\boldsymbol{u}=\sum_{i=1}^{n_{\mathrm{m}}} \boldsymbol{\varphi}_{i} \boldsymbol{q}_{i}=\sum_{i=1}^{n_{\mathrm{m}}} \boldsymbol{\varphi}_{i} \boldsymbol{\Phi}_{s} \boldsymbol{q}^{*}=\sum_{i=1}^{n_{\mathrm{m}}} \boldsymbol{\varphi}_{i}^{*} \boldsymbol{q}^{*}
$$

where $\boldsymbol{\varphi}_{i}$ is the $i$ th orthogonalized Craig-Bampton mode, $n_{\mathrm{m}}$ is the number of modal modes, $\boldsymbol{q}_{i}$ is the modal amplitude of the $i$ th order, and $*$ is the orthogonalized symbol.

Each modal $\varphi_{i}{ }^{*}$ of the orthogonalized CraigBampton modes has an associated inherent frequency $\omega$. A linear elastic body is capable of undergoing large motion, characterized by six nonlinear generalized coordinates for a body coordinate system (BCS). The small, linear elastic deformations of a flexible body relative to this BCS are described by a linear combination of mode shapes.

The governing equations for a multi-body mechanism with multi-topological states are derived from Lagrange's equations:

$$
\begin{aligned}
& \frac{\mathrm{d}}{\mathrm{d} t}\left(\frac{\partial \boldsymbol{L}}{\partial \xi}\right)-\frac{\partial \boldsymbol{L}}{\partial \xi}+\frac{\partial \boldsymbol{F}}{\partial \xi}+\left(\frac{\partial \boldsymbol{\Psi}}{\partial \xi}\right)^{\mathrm{T}} \lambda-\boldsymbol{Q}=0, \\
& \boldsymbol{\Psi}(\xi, t)=0
\end{aligned}
$$

where $\boldsymbol{L}$ represents Lagrangian, $\boldsymbol{F} \in \mathbb{R}^{6 \times k \times n_{\mathrm{s}}}$ represents the energy dissipation function, $\lambda \in \mathbb{R}^{n_{\mathrm{c}}}$ are the Lagrange multipliers for the constraints, $\xi$ represents generalized coordinates of the body, $\boldsymbol{Q} \in \mathbb{R}^{6 \times k \times n_{\mathrm{s}}}$ are the generalized applied forces projected on $\xi$, $\boldsymbol{q} \in \mathbb{R}^{6 \times k \times n_{\mathrm{s}}}$ are modal coordinates, $\boldsymbol{\Psi} \in \mathbb{R}^{n_{\mathrm{c}}}$ are constraint equations about $\xi, n_{\mathrm{c}}$ is the number of constraint equations, $n_{\mathrm{s}}$ is the number of subparts, and $t$ is time.

\section{Dynamic properties and correlated per- formance of multi-DOF rigid-flexible system}

A rigid-flexible multi-body topology-variable mechanism has bodies, joints, and forces. The interconnected relations between subparts are usually represented by mechanism joints: revolute joints, translational joints, cylindrical joints, spherical joints, planar joints, constant-velocity joints, screw joints, fixed joints, and higher pair joints.

The dynamic questions are expressed by differential algebraic equations (DAE), which are 
also called Euler-Lagrange equations. The constraint equations $\boldsymbol{\Psi} \in \mathbb{R}^{n_{\mathrm{c}}}$ can be subdivided by invariable constraints $\boldsymbol{\Psi}_{\text {in }}$ and variable topology constraints $\boldsymbol{\Psi}_{\mathrm{va}}$ according to multiple states of the mechanism.

$$
\boldsymbol{\Psi}=\left(\boldsymbol{\Psi}_{\text {in }}, \boldsymbol{\Psi}_{\text {va }}\right) \in \mathbb{R}^{n_{\mathrm{c}}}
$$

The variable topology mechanism from the $(i-1)$ th state to the $i$ th state is realized by activating the variable topology constraint equations.

$$
\boldsymbol{\Psi}_{i}=(\xi, \dot{\xi}, \ddot{\xi}, t)>0
$$

where $\boldsymbol{\Psi}_{i} \in \mathbb{R}^{n_{\mathrm{t}}}, i=1,2, \cdots, n_{\mathrm{t}}$.

The flexible body equations need stiffly stable, variable-order, variable-step, multi-step implicit integration. The numerical methods for solving the differential Eq. (6) lead to truncation error and round-off error. The residual error is used as the iteration convergence criterion. The ratio between the specified truncation error tolerance and the computed truncation error is used to optimize the iteration step $h$. The Jacobian matrix of constraint equations $\boldsymbol{\Psi}_{\xi}^{\mathrm{T}}$ can be obtained by derivation of $\xi$ through $m$ th-order backward differentiation formula $(m=1)$ :

$$
\begin{gathered}
\left(\boldsymbol{\Psi}_{\xi}\right)_{(i, j)}=\frac{\partial \boldsymbol{\Psi}_{i}}{\partial \xi_{j}} \in \mathbb{R}^{n_{\mathrm{c}} \times n_{\mathrm{s}}}, \\
i=1,2, \cdots, n_{\mathrm{c}}, j=1,2, \cdots, n_{\mathrm{s}} .
\end{gathered}
$$

The matrix condition number measures the asymptotically worst case of how much the function can change in proportion to small changes in the argument. A function with a low condition number is well-conditioned, while a function with a high condition number is ill-conditioned. The first few steps may not converge, and a prohibitively small adaptive iteration step $h$ defined by Courant number is needed initially to maintain stability.

The steps for obtaining the dynamic properties and correlated performance of multi-DOF rigidflexible system are indicated below:
Step 1: Discretize the flexible structure into mesh model and multi-DOF multi-body system;

Step 2: Extract the modal vector expression $\boldsymbol{\Phi}$ using the structure material property, loading, interconnected joints, and DOF constraints $\boldsymbol{\Psi}$ into a file such as modal neutral file (MNF);

Step 3: Reduce scale of the modal vector based on Craig-Bampton modal truncation using Eq. (5);

Step 4: Assemble substructures using a multipoint constraint (MPC) procedure and integrate the reduced modal vector into the rigid-flexible multibody dynamic model using Eq. (6);

Step 5: Pre-define residual error $\varepsilon_{k}$ after $k-1$ iterations, $n$ steady problems, sub-step iteration, step $h$, threshold value $\delta$;

Step 6: Find $i$ with the highest order modal $\phi_{i}$ where $i \in Z^{+}, \quad i \geq 1$, and $\phi_{i} \geq \delta$, if $\phi_{i} \equiv \varnothing$, let $\delta \leftarrow \delta+\varepsilon^{+}$and $h \leftarrow h-\varepsilon^{+}$;

Step 7: Activate the higher order modes by re-reducing the modal vector using Kriging interpolation;

Step 8: After many approximate steady states starting from the initial state, where each steady state has multi-iterations, get the transient state;

Step 9: Stop the iteration till the residual error $\varepsilon_{k}$ meet the requirements by $\operatorname{cond}\left(\boldsymbol{\Psi}_{\xi}\right) \rightarrow 0$;

Step 10: Get the dynamic properties and correlated performance by the generalized coordinates $\xi$.

\section{Correlated performance reinforcing of the multi-actuated mechanism}

Thermal-magnetic breakers provide reliable overload and short-circuit protection for conductors and connected apparatus. A typical LVCB that uses air to extinguish an electric arc is shown in Fig. 2. The short circuit instantaneous or short-time delay interruption is triggered by an electromagnetic force $F_{\mathrm{em}}$ actuated from an electromagnet-drawbar-jump buckle mechanism. The over-load long-time delay is triggered by a thermobimetallic strip force $F_{\mathrm{bi}}$ actuated by a thermobimetallic strip-drawbar mechanism. The electrodynamic force $F_{\text {ed }}$ applied to the moving contactor overcomes the force of the contact spring. Triggering of the drawbar leads to the latch buckle $H T$ 
disconnecting. Concurrently, a metamorphic mechanism occurs resulting from increasing DOFs and spring stored energy. When the upper linkage $B D$ and the lower linkage $A B$ are both at the bottom dead center (BDC) position, the applied force is straight along its axis. It means no torque can be applied and the $K$ then completely connects.

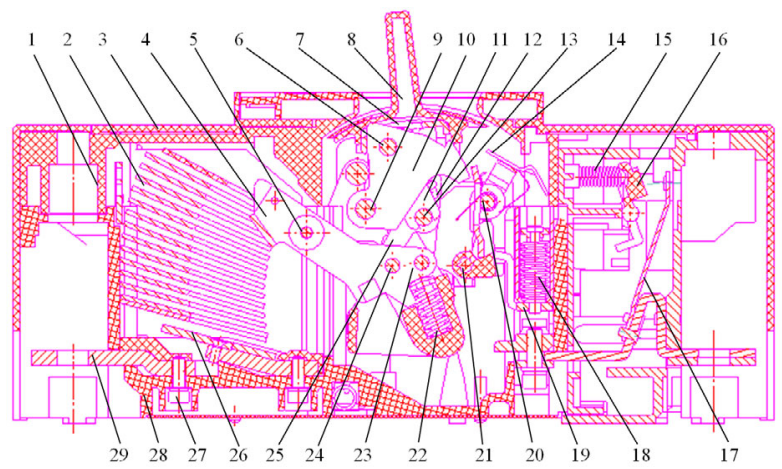

1: wiring terminal; 2: deion plates for arc extinguishing; 3 : upper moulded case; 4 : moving contactor $K$; 5 : fixed joint in moving contactor; 6 : revolute joint $D ; 7$ : one end $J$ of main spring $J B$; 8: manual operating handle $J B$; 9: revolute joint $C$ (ground); 10: jump buckle; 11: upper linkage $B D ; 12$ : revolute joint $O$ (ground); 13 : revolute joint $B ; 14$ : high contact joint $T$ (latch buckle $T H$ ); 15: drawbar spring; 16: thermopretightening regulator; 17: cantilevered thermobimetallic strip; 18: flapped electromagnet armature; 19: short (long time delay tripping trigger); 20: revolute joint $H$ (ground); 21: instantaneous tripping trigger; 22: moving contactor spring; 23: revolute joint $R$ of main shaft (ground); 24: revolute joint $A$; 25: lower linkage $A B ; 26$ : static contactor; 27: fastening bolt; 28 : lower moulded case; 29 : conductor terminal

(a)

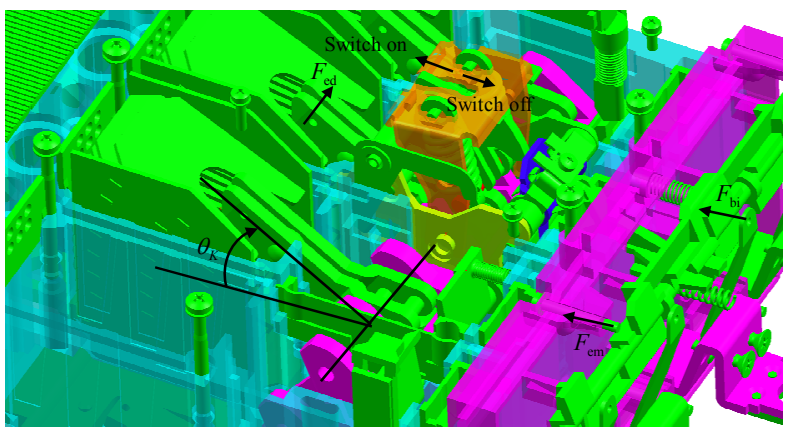

(b)

Fig. 2 Parts and joints of LVCB where the activating mechanism is decomposed into three submechanisms: the main switch connector mechanism, the electromagnetdrawbar-jump buckle mechanism, and the bimetallic strip-drawbar mechanism, which are described by electrodynamic force $F_{\text {ed }}$, electromagnet force $F_{\text {em }}$, and bimetallic strip force $\boldsymbol{F}_{\mathrm{bi}}$, respectively

(a) Cutaway view of rigid-flexible multi-body dynamics mechanism; (b) Isometric drawing of rigid-flexible multibody dynamics mechanism
The flapped electromagnetic system comprises a coil and a gag bit component. The attraction force of the electromagnet trip mechanism $F_{\text {em }}$ (Fig. 2b) can be obtained by Maxwell's formula and Ampere's circuital law:

$$
F_{\text {em }}=\frac{\boldsymbol{B}^{2} S}{2 \mu_{0}}
$$

where $\boldsymbol{B}$ represents magnetic flux density, $S$ represents the cross sectional area of electromagnetic iron core, and the permeability of the vacuum $\mu_{0}$ is $4 \pi \times 10^{-7} \mathrm{~N} / \mathrm{A}^{2}$.

Thermobimetallic elements (TBEs) are the basic elements of thermal circuit breakers (CBs) of automatic release devices (ARDs) and electrothermal current relays (ECRs) intended for protection of electric installations during current over loads. The cantilevered thermobimetallic strip is shown in Fig. 3.

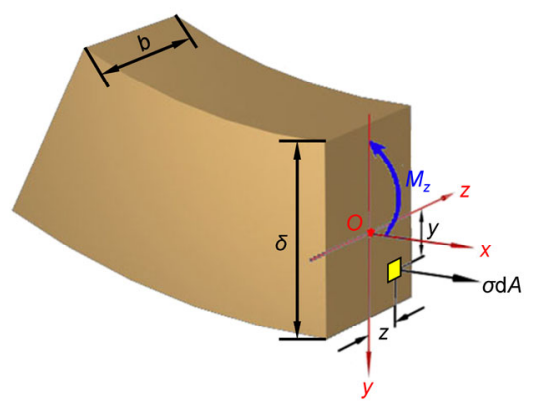

Fig. 3 Cantilevered thermobimetallic strip of the LVCB

The pushing force $F_{\text {bi }}$ (Fig. 2b) can be obtained from the thermodynamic energy equation:

$$
\begin{gathered}
m c \frac{\mathrm{d} \Delta T}{\mathrm{~d} t}+k_{\mathrm{T}} S \Delta T=i^{2} R, \\
F_{\mathrm{bi}}=\frac{k b \delta^{2} E}{4 l} \Delta T,
\end{gathered}
$$

where $m$ is the mass of the thermobimetallic strip; $k_{\mathrm{T}}$ is the surface heat transfer coefficient; $S$ is the surface area; $i$ is the actual current; $R$ represents the electrical resistance; $c$ represents the specific heat capacity; $k$ is the specific thermal deflection; $b$ is the width of the bimetallic strip; $\delta$ is the thickness of the bimetallic strip; $l$ is the length of the bimetallic strip; $E$ is the 
Young's modulus of elasticity; $\Delta T$ is the temperature variation.

For solid rectangular section of thermobimetallic strip, moment of inertia $I_{z}$ of the neutral axis $z$ and section modulus in bending $W_{z}$ of the neutral axis $z$ are

$$
I_{z}=\frac{b \delta^{3}}{12}, W_{z}=\frac{b \delta^{2}}{6} .
$$

Using the Hooke strain theorem, for purebending cantilever beam under bending moment $M$, the bending normal stress $\sigma$ and maximum bending normal stress $\sigma_{\max }$, substituted into Eq. (12), are

$$
\sigma=\frac{M y}{I_{z}}, \sigma_{\max }=\frac{M}{W_{z}}=\frac{6 F_{\mathrm{bi}} l}{b \delta^{2}} .
$$

The electrodynamic repulsion force (Kawase et al., 1997) $F_{\text {ed }}$ (Fig. 2b) contains the Holm force $F_{\text {Holm }}$ and the Lorentz force $F_{\text {Lorentz: }}$ :

$$
F_{\text {ed }}=F_{\text {Holm }}+F_{\text {Lorentz }} \text {. }
$$

The pushing force of bimetallic strip $F_{\mathrm{bi}}$ and the electrodynamic repulsion force $F_{\text {ed }}$ do not coexist. The contact area is far smaller than the contact surface area because the contact surface is rough in microscopic scales. Then, the current elements shrink at the contact point, which leads to a repulsion force. The Holm force $F_{\text {Holm }}$ is caused by current constriction and a skin effect which exists only during the contact process of the main switch connector $K$.

$$
F_{\text {Holm }}=\frac{\mu_{0} i^{2}}{4 \pi} \ln \sqrt{\frac{S}{S_{0}}}=\frac{\mu_{0} i^{2}}{4 \pi} \ln \sqrt{\frac{c_{K} H_{\mathrm{B}} S}{F_{K}}},
$$

where $S$ is the cross sectional area of contact point $K$, and $S_{0}$ is the effective contact area. For Ag alloy electrical contact material CAgw50, $c_{K} \in[0.3,1]$ is the contact coefficient, $H_{\mathrm{B}}$ is the Brinell hardness of the contact point, and $F_{K}$ is the contact force of contact point $K$.

The Lorentz force $F_{\text {Lorentz }}$ can be obtained from the Biot-Savart law which applies until the electric arc is extinguished. $F_{\text {Lorentz }}$ depends on the current and contact angle of $K$.

$$
F_{\text {Lorentz }}=\int_{\text {contact }}(\boldsymbol{J} \times \boldsymbol{B}) \mathrm{d} V,
$$

where $\boldsymbol{J}$ represents the electric current density, and $V$ represents volume.

$$
\begin{gathered}
\boldsymbol{J}=\operatorname{rot} \boldsymbol{T}, \\
\operatorname{rot}((1 / R) \boldsymbol{J})=0, \\
\operatorname{div} \boldsymbol{J}=0, \\
\oint_{s} \boldsymbol{T} \cdot \mathrm{d} l=i,
\end{gathered}
$$

where $\boldsymbol{T}$ represents the current vector, rot represents curl, and div represents divergence.

The magnetic flux density $\boldsymbol{B}$ can be obtained using the electric current density $\boldsymbol{J}$ :

$$
\begin{gathered}
\boldsymbol{B}=\operatorname{rot} \boldsymbol{A}, \\
\operatorname{rot}((1 / \mu) \operatorname{rot} \boldsymbol{A})=\boldsymbol{J},
\end{gathered}
$$

where $\mu$ is the magnetic conductivity.

The interruption necessarily causes an arc, while switching on potentially causes an arc. The arc energy $\int i^{2} \mathrm{~d} t$ is due to tripping peaks when the short circuit occurs. When tripping occurs, using Kirchhoff's circuit laws, specifically the Kirchhoff voltage law, the voltage balance equation can be built. The arc energy causes arc erosion of the contactor and a rise in the temperature of the conductor terminal $T_{\text {conductor }}$, case $T_{\text {case, }}$ and operating handle $T_{\text {handle. }}$ The temperature variation $\Delta T$ of the $L V C B$ during operation is attributed mainly to resistance loss, eddy current loss, hysteresis loss, and dielectric loss.

\section{Correlated performance reinforcing of a typical LVCB}

Let $\theta_{K}$ (Fig. 2b) stand for the opening angle of the main switch connector $K$. The damp of the main spring, moving contactor spring, and drawbar spring is $1.1 \times 10^{-4} \mathrm{~N} \cdot \mathrm{s} / \mathrm{mm}$. Let the electrical resistivity of the moving and static contactors $R=3 \times 10^{-7} \Omega \cdot \mathrm{m}$. The relative magnetic permeability $\mu_{\mathrm{r}}=1000$, the shortcircuit current (SCC) is set as $20 \mathrm{kA}, \xi=0.46, F_{K}=$ $19 \mathrm{~N}$, and $H_{\mathrm{B}}=900 \mathrm{~N} / \mathrm{mm}^{2}$. The correlated tripping 
performance using Eq. (6) is shown in Fig. 4 where $k_{\text {main }}=14.4 \mathrm{~N} / \mathrm{mm}$.

From Fig. 4 and Table 1, the moving connector $K$ shows springback vibration during the interruption process. This can be attributed mainly to the stiffness and damp of the main spring $k_{\text {main }}$ and $\xi_{\text {main }}$, moving contactor spring $k_{\text {conductor }}$ and $\xi_{\text {contactor, }}$, and drawbar spring $k_{\text {drawbar }}$ and $\xi_{\text {drawbar. }}$. The maximum opening angle and maximum opening resultant displacement of $K$ are increased to eliminate electric arc re-ignition. Using analogical design methodology with and without structure flexibility, the average absolute opening resultant velocity decreases when structure flexibility is considered. The simulation showed that the tripping frequencies of the key structures of the LVCB were in descending order: jump buckle>latch buckle>lower linkage> upper linkage.

\section{Electrical performance experiments of LVCB}

The subpart flexibility properties are difficult to measure. Therefore, the design of experiments method is employed by comparing and analogizing the relative flexibility of the subparts. The hardmeasuring properties are revealed by correlated relatively easy-measuring parameters, such as temperature. The trip mechanism was validated by an electrical performance experiment of LVCB which is shown in Figs. 5 and 6. The experimental LVCB has three phases. If the $\mathrm{LVCB}$ remains $\mathrm{ON}$ at the rated current, the stable temperature rise of the LVCB should be less than $1 \mathrm{~K}$ after $1 \mathrm{~h}$. The measurement points of the LVCB included mainly the conductor terminal temperature $T_{\text {conductor, }}$, case temperature $T_{\text {case }}$, and operating handle temperature $T_{\text {handle }}$. The trip mechanism should trip reliably at ambient temperatures ranging from $-20^{\circ} \mathrm{C}$ to $85^{\circ} \mathrm{C}$. The trip angle of moving connector $K$ of LVCB was obtained using an angular displacement sensor. Optical fiber thermistor temperature sensors have the advantage of resistance to electromagnetic interference.

Results of the temperature rise experiments are shown in Table 2.

Table 2 shows that the jump buckle joint was the component most vulnerable to abrasion due to its high tripping frequency and velocity, followed by the latch
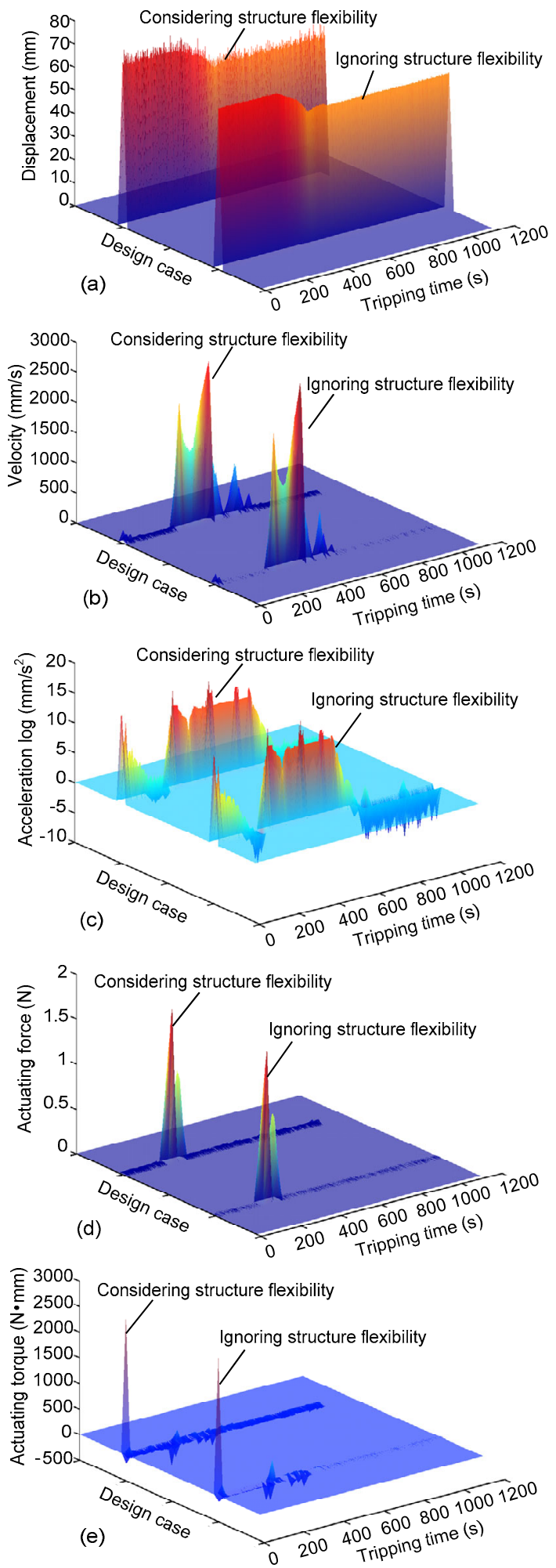

Fig. 4 Displacement, velocity, acceleration, force, and torque on $K$ by correlated performance reinforcing (a) Displacement $S_{K}$ of moving connector $K$; (b) Velocity $v_{K}$ of moving connector $K$; (c) Acceleration $a_{K}$ of moving connector $K$; (d) Repulsion force $F_{\text {ed }}$ on moving connector $K$; (e) Torque $M_{K}$ applied to moving connector $K$ 
Table 1 Trip performance of the LVCB with and without structure flexibility

\begin{tabular}{|c|c|c|c|c|c|c|}
\hline \multicolumn{2}{|c|}{ Item } & $\begin{array}{c}\text { Displacement, } \\
S_{K}(\mathrm{~mm})\end{array}$ & $\begin{array}{c}\text { Velocity, } \\
v_{K}(\mathrm{~mm} / \mathrm{s})\end{array}$ & $\begin{array}{l}\text { Acceleration, } \\
a_{K}\left(\mathrm{~mm} / \mathrm{s}^{2}\right)\end{array}$ & $\begin{array}{c}\text { Repulsion force, } \\
F_{\text {ed }}(\mathrm{N})\end{array}$ & $\begin{array}{c}\text { Torque, } \\
M_{K}(\mathrm{~N} \cdot \mathrm{mm})\end{array}$ \\
\hline \multirow{2}{*}{$\begin{array}{l}\text { Ignoring } \\
\text { structure } \\
\text { flexibility }\end{array}$} & Mean & 61.89 & 321.48 & $2.45 \times 10^{5}$ & 0.086 & 4.48 \\
\hline & Maximum & 69.17 & $2.97 \times 10^{3}$ & $2.85 \times 10^{7}$ & 1.705 & $2.71 \times 10^{3}$ \\
\hline \multirow{2}{*}{$\begin{array}{l}\text { Considering } \\
\text { structure } \\
\text { flexibility }\end{array}$} & Mean & 62.42 & 287.38 & $2.72 \times 10^{5}$ & 0.096 & 5.99 \\
\hline & Maximum & 69.17 & $2.59 \times 10^{3}$ & $248 \times 10^{7}$ & 1.713 & $2.71 \times 10^{3}$ \\
\hline
\end{tabular}

Table 2 Temperature rise of different circuit breaker components of electrical performance experiments of circuit breaker

\begin{tabular}{lcccccc}
\hline \multirow{2}{*}{ Components with temperature rise } & \multicolumn{5}{c}{ Temperature rise at different time progress $\left({ }^{\circ} \mathrm{C}\right)$} \\
\cline { 2 - 6 } & $5 \%$ & $10 \%$ & $20 \%$ & $40 \%$ & $60 \%$ & $100 \%$ \\
\hline Jump buckle joint (Label 10 in Fig. 2a) & 1.21 & 1.46 & 2.55 & 6.05 & 7.38 & 8.31 \\
Latch buckle joint, high contact (Label 14 in Fig. 2a) & 1.09 & 1.18 & 2.34 & 5.45 & 6.65 & 7.49 \\
Lower linkage joint (Label 25 in Fig. 2a) & 1.06 & 1.12 & 2.25 & 5.33 & 6.47 & 7.38 \\
Upper linkage joint (Label 11 in Fig. 2a) & 0.98 & 0.96 & 2.07 & 4.92 & 5.99 & 6.73 \\
Moving contactor joint (Label 4 in Fig. 2a) & 0.67 & 0.43 & 1.41 & 3.35 & 4.09 & 4.63 \\
Fixed contactor joint, high contact (Label 5 in Fig. 2a) & 0.64 & 0.41 & 1.35 & 3.21 & 3.91 & 4.42 \\
Deion plates for arc extinguishing (Label 2 in Fig. 2a) & 0.60 & 0.36 & 1.28 & 3.02 & 3.66 & 4.12 \\
Cantilevered thermobimetallic strip (Label 17 in Fig. 2a) & 0.59 & 0.35 & 1.24 & 2.95 & 3.60 & 4.05 \\
Flapped electromagnet armature (Label 18 in Fig. 2a) & 0.58 & 0.34 & 1.22 & 2.80 & 3.53 & 3.97 \\
\hline
\end{tabular}

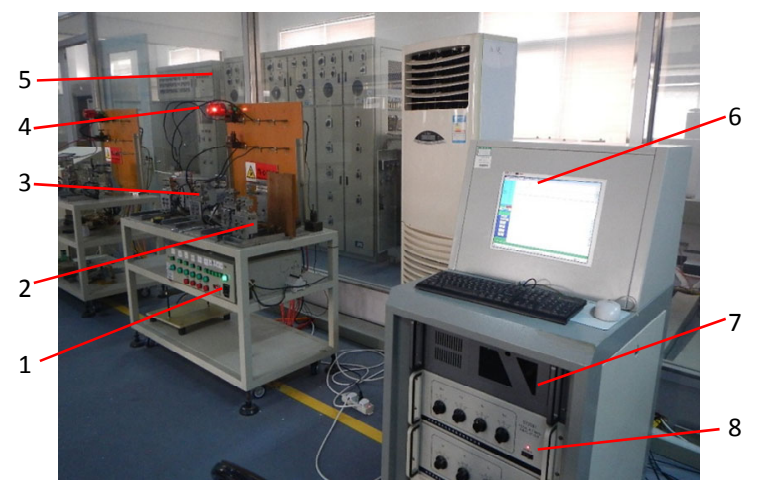

1: control cabinet; 2: operating mechanism; 3: circuit breaker undergoing testing with angular displacement sensor and optical fiber thermistor temperature sensors; 4: alarm lamp; 5 : impedance cabinet used for loading; 6: user interface; 7: signal processor; 8: range transform amplifier

\section{Fig. 5 Electrical performance experiments of LVCB}

buckle joint, lower linkage joint, upper linkage joint, moving contactor joint, and fixed contactor joint. The experimental results are in agreement with the simulation results in terms of the relative extent to which the key structures of the LVCB in temperature rising. For the multi-actuated mechanism, the temperature rise of the flapped electromagnet armature, which is for instantaneous large overload current, was greater

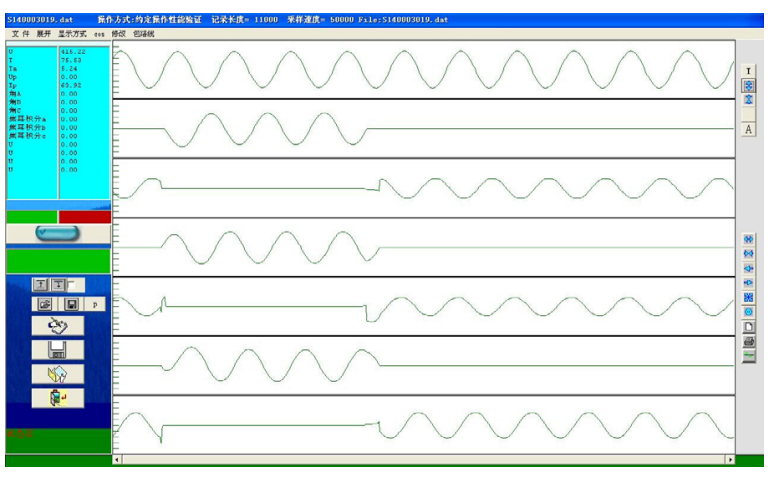

Fig. 6 User interface of electrical performance experiments of LVCB

than that of the cantilevered thermobimetallic strip, which is for small current overloads of long duration.

From the temperature rise experiment results, the peak voltage was $447.7 \mathrm{~V}$, make-break time was $10.58 \mathrm{~ms}$, arcing time was $8.475 \mathrm{~ms}$, Joule integral was $4.631 \mathrm{kA}^{2} \cdot \mathrm{s}$, and the peak current was $1.031 \mathrm{kA}$. The repeatability deviation between the OFF and ON positions of the LVCB contact was improved from $0.024 \mathrm{~mm}$ to $0.016 \mathrm{~mm}$ to reinforce trip performance. The average temperature rise decreased from $7.59{ }^{\circ} \mathrm{C}$ to $5.68{ }^{\circ} \mathrm{C}$ after $100 \%$ time progress tests. 


\section{Conclusions}

1. A multi-actuated mechanism design method considering structure flexibility using correlated performance reinforcing is put forward. Using analogical design methodology with and without structure flexibility, the extent of the performance impact of each subpart can be obtained. The temperature rise analysis is used to reveal structure flexibility properties by evaluating the correlated relatively easymeasuring temperatures.

2. The correlated performance of electromechanical coupling systems can be obtained precisely using the electromechanical correlated method. The correlated trip performance is trapped using adaptive step iteration. Simulation showed that the structure flexibility can reduce the tripping velocity, which is non-negligible, especially for high frequency tripping.

3. Electrical performance experiments using optical fiber thermistor temperature sensors were used to verify the flexibility properties of thermalmagnetic trip mechanism. The experimental results were in agreement with the simulation results about the relative influence extent on temperature rising of the key structures.

\section{References}

Braun, D.J., Goldfarb, M., 2012. Simulation of constrained mechanical systems-part II: explicit numerical integration. Journal of Applied Mechanics, 79(4):041018. [doi:10. $1115 / 1.4005573]$

Deb, M., Sen, D., 2013. Parametric study of the behavior of double toggle switching mechanisms. Mechanism and Machine Theory, 63:8-27. [doi:10.1016/j.mechmachtheory. 2012.12.005]

Hesse, H., Palacios, R., 2012. Consistent structural linearisation in flexible-body dynamics with large rigid-body motion. Computers \& Structures, 110-111:1-14. [doi:10. 1016/j.compstruc.2012.05.011]

Kawase, Y., Mori, H., Ito, S., 1997. 3-D element analysis of electrodynamics repulsion forces in stationary electric contacts taking into account asymmetric shape. IEEE Transactions on Magnetics, 33(2):1994-1999. [doi:10. 1109/20.582692]

Kim, J.G., Lee, K.H., Lee, P.S., 2014. Estimating relative eigenvalue errors in the Craig-Bampton method. Computers \& Structures, 139:54-64. [doi:10.1016/j.compstruc. 2014.04.008]

Lee, J.C., Kim, Y.J., 2006. Effects of nozzle shape on the interruption performance of thermal puffer-type gas circuit breakers. Vacuum, 80(6):599-603. [doi:10.1016/j. vacuum.2005.10.004]

Li, X., Chen, D., Wang, Y., et al., 2007. Analysis of the interruption process of molded case circuit breakers. IEEE Transactions on Components and Packaging Technologies, 30(3):375-382. [doi:10.1109/TCAPT.2007.900051]

Lu, J.G., Du, T.H., Luo, Y.Y., 2007. Study on the instantaneous protection reliability of low voltage circuit breakers. Journal of Zhejiang University-SCIENCE A, 8(3):370377. [doi:10.1631/jzus.2007.A0370]

Machado, M., Moreira, P., Flores, P., et al., 2012. Compliant contact force models in multibody dynamics: evolution of the Hertz contact theory. Mechanism and Machine Theory, 53:99-121. [doi:10.1016/j.mechmachtheory.2012.02. 010]

Nikravesh, P.E., 2007. Initial condition correction in multibody dynamics. Multibody System Dynamics, 18(1):107115. [doi:10.1007/s11044-007-9069-z]

Nossov, V.V., Hage, B., Jusselin, B., et al., 2007. Simulation of the thermal radiation effect of an arc on polymer walls in low-voltage circuit breakers. Technical Physics, 52(5): 651-659. [doi:10.1134/S1063784207050180]

Razi-Kazemi, A.A., 2015. Applicability of auxiliary contacts in circuit breaker online condition assessment. Electric Power Systems Research, 128:53-59. [doi:10.1016/j.epsr. 2015.06.021]

Xu, J.H., Zhang, S.Y., Tan, J.R., et al., 2013a. Collisionless tool orientation smoothing above blade stream surface using NURBS envelope. Journal of Zhejiang UniversitySCIENCE A (Applied Physics \& Engineering), 14(3): 187-197. [doi:10.1631/jzus.A1200160]

Xu, J.H., Zhang, S.Y., Zhao, Z., et al., 2013b. Metamorphic manipulating mechanism design for MCCB using index reduced iteration. Chinese Journal of Mechanical Engineering, 26(2):232-241. [doi:10.3901/CJME.2013.02. 232]

\section{中文概要}

题 目: 考虑结构柔性的多激励机构关联性能强化设计 方法

目 的：揭示多场耦合环境下的多自由度系统中柔性结构 对机构性能的影响规律, 以低压断路器多激励操 动机构为例, 研究机-电-热-磁耦合的多模态分断 性能强化设计方法, 提高短路、过载、欠压、缺 相和漏电时的瞬时、短延时和长延时分断性能。

创新点: 1 . 将多自由度刚柔耦合系统的物理坐标转换为模 态坐标, 实现模型降阶和关联性能解耦; 2. 运用 类比法获得零部件对关联性能的影响度, 通过实 验设计将难测属性转化为关联易测量, 实现机构 的关联性能强化。 
方 法：1. 将多自由度的含柔体动力学系统转化为自由度 乘模态阶的方阵, 通过 Craig-Bampton 逐层降阶 模态矩阵, 并由迭代步反馈激活柔体的高阶模 态; 2 . 通过类比求解霍尔姆力、洛伦兹力、电动 斥力、电磁力和悬臂双金属片激励力, 获得多激 励系统机构关节的性能关联程度; 3. 通过低压断 路器电气实验和温升实验, 验证零件结构柔性对 机构的性能影响规律, 实现多激励机构多模态分 断性能强化设计。
结 论: 1. 提出的考虑结构柔性的多激励机构关联性能强 化设计方法有助于提高多自由度动力学系统的 瞬态性能; 2. 结构柔性降低分断性能, 使高频分 断中的峰值段性能退化; 3. 基于实验设计的电气 实验和温升实验, 验证了多激励机构关联性能强 化方法的有效性。

关键词: 多激励机构; 机-电-热-磁耦合; 多体刚柔耦合动 力学系统; 低压断路器; 关联性能强化 